\title{
3-S36-1 Symposium36
}

\section{An exciting future of pharmacology}

\section{Mitsuhiro Yoshioka}

\section{Dept. Neuropharmacol. Hokkaido Univ.}

Pharmacology is an energetic area of biomedical science, linking together chemistry, physiology and pathology, and crucial for discovering new drugs to help fight diseases. For the development of new drugs, advances in basic science must be achieved to create the seeds of new drugs. Such seeds must be translated into clinical trials at bedside. Thus, pharmacology requires the multidisciplinary integration of various levels of studies from bench to bedside and demands that we utilize a wide spectrum of methods to achieve our final goal. Pharmacology has adopted the latest developed technology immediately and raised the precision of not only the studies but also experimental therapeutics. We should discuss about the new exciting technique that is applied to a pharmacological study. 